INFLAMMATORY BOWEL DISEASE

\title{
Oral contrast enhanced bowel ultrasonography in the assessment of small intestine Crohn's disease. A prospective comparison with conventional ultrasound, $x$ ray studies, and ileocolonoscopy
}

\author{
F Parente, S Greco, M Molteni, A Anderloni, G M Sampietro, P G Danelli, R Bianco, S Gallus, \\ G Bianchi Porro
}

Gut 2004;53:1652-1657. doi: 10.1136/gut.2004.041038

\begin{abstract}
Background/Aim: Although ultrasound (US) has proved to be useful in intestinal diseases, barium enteroclysis (BE) remains the gold standard technique for assessing patients with small bowel Crohn's disease (CD). The ingestion of anechoic non-absorbable solutions has been recently proposed in order to distend intestinal loops and improve small bowel visualisation. The authors' aim was to evaluate the accuracy of oral contrast US in finding CD lesions, assessing their extent within the bowel, and detecting luminal complications, compared with BE and ileocolonoscopy.

Methods: 102 consecutive patients with proven CD, having undergone complete $x$ ray and endoscopic evaluation, were enrolled in the study. Each US examination, before and after the ingestion of a polyethylene glycol (PEG) solution $(500-800 \mathrm{ml})$, was performed independently by two sonographers unaware of the results of other diagnostic procedures. The accuracy of conventional and contrast enhanced US in detecting CD lesions and luminal complications, as well as the extent of bowel involvement, were determined. Interobserver agreement between sonographers with both US techniques was also estimated.

Results: After oral contrast, satisfactory distension of the intestinal lumen was obtained in all patients, with a mean time to reach the terminal ileum of 31.4 (SD 10.9) minutes. Overall sensitivity of conventional and oral contrast US in detecting CD lesions were $91.4 \%$ and $96.1 \%$, respectively. The correlation coefficient between US and $x$ ray extent of ileal disease was $r_{1}=0.83(p<0.001)$ before and $r_{2}=0.94(p<0.001)$ after PEG ingestion; $r_{1}$ versus $r_{2} p<0.01$. Sensitivity in detecting strictures was $74 \%$ for conventional US and $89 \%$ for contrast US. Overall interobserver agreement for bowel wall thickness and disease location within the small bowel was already good before but significantly improved after PEG ingestion.

Conclusions: Oral contrast bowel US is comparable with BE in defining anatomic location and extension of $C D$ and superior to conventional US in detecting luminal complications, as well as reducing interobserver variability between sonographers. It may be therefore regarded as the first imaging procedure in the diagnostic work up and follow up of small intestine CD.
\end{abstract}

See end of article for authors' affiliations

Correspondence to:

Dr F Parente, Department and Chair of

Gastroenterology, L Sacco University Hospital, Milan, Italy; fabrizio.parente@ tiscalinet.it

Accepted for publication 27 April 2004 in

the last decade bowel ultrasound (US) has gained importance as a non-invasive and reliable imaging modality in the diagnosis and follow up of Crohn's disease (CD), allowing clear visualisation of the involved bowel segments and surrounding mesentery. ${ }^{1-6}$ Nonetheless, invasive procedures such as barium enteroclysis (BE) are still largely used in order to provide optimal visualisation of luminal surface pattern, to evaluate the exact extension of disease within the bowel, as well as to reveal intestinal complications like strictures and fistulas. ${ }^{7-9}$

Recent reports suggest that the ingestion of an iso-osmolar polyethylene glycol (PEG) solution improves visualisation of small bowel loops at US giving results comparable with BE in several intestinal disorders, including coeliac disease and CD. ${ }^{10-13}$ To date, however, no large prospective study has specifically compared this new US technique with conventional bowel US, $x$ ray studies, and ileocolonoscopy in the diagnostic work up of small bowel CD. Moreover, the potential ability of this procedure in improving agreement between sonographers on the site and extension of CD lesions by reducing air artefacts (which hinder sonographic reflection) and by favouring dissociation, and thus visualisation, of one intestinal loop from another remains to be fully determined.
The primary aim of this study was therefore to investigate, in a large series of consecutive patients with small bowel CD, the accuracy of oral contrast US in locating the site of $\mathrm{CD}$ lesions, their extension within the small bowel, as well as the presence of luminal complications compared with conventional bowel US, using BE and ileocolonoscopy as gold standards. The study also aimed to determine the interobserver variability between sonographers of this new technique in comparison with that of conventional bowel US.

\section{MATERIAL AND METHODS}

\section{Patients}

Consecutive patients with proven small bowel CD seen in the outpatient IBD clinic or admitted to the Department of Gastroenterology of L Sacco University Hospital between December 2002 and July 2003 were invited to take part in this prospective study. Diagnosis of CD had been achieved in all patients using standard methods. ${ }^{14}$ The criteria for admission

Abbreviations: $\mathrm{BE}$, barium enteroclysis; $\mathrm{BWT}$, bowel wall thickness; $C D$, Crohn's disease; CT, computed tomography; MRI, magnetic resonance imaging; NPV, negative predictive value; PEG, polyethylene glycol; PPV, positive predictive value; US, ultrasound. 
to the study also required that patients were over 18 years of age, had an adequate medical records of clinical, anatomical, and therapeutic characteristics of disease, and had to be submitted to colonoscopy (or barium enema) and BE for disease restaging. Patients were excluded from the study if they had only anorectal, gastroduodenal, or colonic localisation of the disease. Exclusion criteria also included pregnancy, renal insufficiency (serum creatinine levels of $2.5 \mathrm{mg} / \mathrm{dl}$ or greater), as well as severe clinical conditions (that is, CD patients with acute intestinal obstruction or sepsis).

\section{Methods \\ Protocol of the study}

Each patient was initially submitted to a standardised clinical interview and physical examination by a gastroenterologist who did not take part in the imaging procedures but requested those laboratory investigations deemed necessary. In particular, clinical details were carefully sought concerning duration of disease, initial diagnosis, previous surgery, number of recurrences, as well as current and previous medical treatments. Disease activity was assessed on admission to the study using the CD activity index (CDAI) ${ }^{15}$. Each patient underwent conventional and oral contrast bowel US in the same day as the first diagnostic procedure. BE and colonoscopy (and/or barium enema) were subsequently performed on different days. All patients were independently evaluated, both at conventional and contrast enhanced US (see below), by two sonographers who had an experience exceeding $>4000$ (FP) and >2000 (SG) sonographic examinations of the bowel, respectively.

\section{Conventional US}

Sonographic scan of the abdomen was carried out with a real time ultrasonic apparatus (Aloka Prosound 5000 or Hitachi 6500 ) initially using a convex 3.5-6 MHz transducer, and then, for a detailed examination, a linear 7.5-10.0 $\mathrm{MHz}$ probe. Patients were examined after an overnight fast but without any special preparation. As previously reported, ${ }^{16}$ intestinal wall thickness $\geqslant 4 \mathrm{~mm}$ (measured from the edge of the outer wall to the inner echogenic mucosa-gas acoustic surface) was considered pathological, provided that it could be measured both in longitudinal and transverse sections and it could be reproduced for at least $4 \mathrm{~cm}$ in length.

In patients in whom more than one segment was involved, the maximum bowel wall thickness (BWT) was used. Abdominal complications detectable at US such as stenosis, internal fistulas, and abscesses were specifically looked for and recorded as described elsewhere. ${ }^{17}$ In particular, strictures were considered present when US revealed coexistence of thickened and stiff bowel wall, narrowing of the intestinal lumen together with distended fluid, or echogenic content filled loop just above the stiffened bowel segment. The anatomical region where abnormal findings were localised was classified according to a standardised form which included seven sections: duodenum/jejunum, ileum, caecum/ascending colon, transverse colon, sigmoid/descending colon, or rectum, even if left sided lesions were not specifically considered in this report.

At the end of this plain preliminary study two independent reports were prepared by sonographers. Elasticity of affected bowel segments was assessed by evaluating the presence of intermittent variation in loop profile and luminal diameter (registered for a 10 minute period of observation) as well as variation in BWT during transabdominal compression; an elasticity score was assigned according to an arbitrary 1-3 scale where 1 stands for a stiffened loop with lack of peristalsys, 2 for a segment showing reduced motility and compressibility, and 3 for an easily compressible loop with almost normal motor activity.

\section{Oral contrast US}

Immediately after conventional US, the patient was invited to drink the contrast solution, which was freshly prepared by dissolving in $1000 \mathrm{ml}$ of tap water, a granular powder containing PEG $335059.0 \mathrm{~g}$, anhydrous sodium sulfate $5.68 \mathrm{~g}$, sodium bicarbonate $1.68 \mathrm{~g}$, sodium chloride $1.46 \mathrm{~g}$, and potassium chloride $0.74 \mathrm{~g}$ (Klean-prep, Norgine, Milan, Italy). The osmolality of this solution is $280-290 \mathrm{mOsm} / \mathrm{kg}$, as determined by the freezing point method. Increasing aliquots of the iso-osmolar oral contrast up to a maximum of $800 \mathrm{ml}$ were drunk progressively by each patient in order to obtain a good distension of the entire small bowel lumen. Based on previous reports ${ }^{12}{ }^{13}$ and a unpublished personal series, a minimum of $500 \mathrm{ml}$ of PEG solution was deemed necessary to completely dissociate one intestinal loop from another and visualise the intestinal surface. US sections of the bowel, with the patient in the supine position, were done 10 minutes after PEG ingestion and then repeated at 10 minute intervals until the contrast was seen to flow through the terminal ileum into the caecum, when a retrograde US examination of the small bowel was performed. At the end of this post distension study two new US reports were prepared independently by each sonographer. During contrast studies, elasticity of affected bowel segments was assessed according to the degree of luminal distension which was defined as separation of the two luminal surfaces by contrast material without collapse. In particular, sonographers assigned an arbitrary score based on estimate of wall distension: stiff loops were assigned a distension score of 1 , moderate distended loops received a score of 2, adequately distended loops received a distension score of 3. Post contrast distensibility score was compared with the precontrast elasticity score determined by the same sonographer (see above).

\section{$X$ ray studies}

$\mathrm{BE}$ was performed by a radiologist with specific experience in this type of examination who was unaware of the results of bowel US using the conventional technique after transnasal jejunal intubation. ${ }^{19}$ Small bowel abnormalities with particular reference to disease length (expressed in $\mathrm{cm}$ ), number and site of strictures, and fistulas were written at the end of examination on a specific report. Patients in whom the examination was not feasible (that is, unable to pass the nasal tube into the distal duodenum or jejunum) were excluded from the study. Computed tomography (CT) scan, after the ingestion of an oral contrast medium (Gastromiro, Bracco, Milan, Italy) followed by intravenous injection of $150 \mathrm{ml}$ contrast agent (Ultravist 300, Schering, Berlin, Germany) for contrast CT evaluation was performed whenever clinically indicated (that is, when abdominal abscesses were suspected). Patients whose ileocolonic anastomosis was intraversable at endoscopy also underwent conventional double contrast barium enema, which was taken into consideration as a reference standard for colon morphology and ileocolonic stenosis.

\section{Ileocolonoscopy}

All patients underwent colonoscopy by experienced endoscopists under sedation with intravenous midazolam (Roche, Milan, Italy) and petidine (Molteni, Florence, Italy). In all patients intubation of the terminal ileum or neoterminal ileum (in those with ileocolonic anastomosis) was attempted.

\section{Surgical findings}

In patients undergoing surgery for CD complications, surgical findings were used as the gold standard to assess internal fistulas and intra-abdominal abscesses in comparison with the results of the diagnostic techniques. Definitions and classification of fistulas and abscesses were made in 
accordance with the existing literature and previous studies of ours. ${ }^{618}$ The study was approved by the L Sacco Hospital Ethics Committee and written informed consent was obtained from all patients before entering the study.

\section{Statistical analysis}

To estimate the interobserver agreement, and the agreement between the two types of US and the final diagnosis in identifying the sites of CD lesions, we considered the simple kappa coefficient. To obtain the accuracy of US in detecting $\mathrm{CD}$ lesions along the bowel as well as strictures/fistulas, we calculated the sensitivity and specificity: sensitivity was defined as the proportion of patients with a detection at US divided by the patients with CD lesions, strictures/fistulas as final diagnosis; specificity was defined as the proportion of patients with a negative detection at US divided by the patients without CD lesions, strictures/fistulas. Finally, the correlation between the radiographic and US extent of small bowel CD (the latter measured both at conventional examination and after oral contrast) was assessed by Pearson's correlation coefficient. The exact $95 \%$ confidence interval (CI) for each statistic was calculated from the binomial distribution. Elasticity scores as determined by the two operators were compared between the two US protocols (conventional and contrast enhanced US) by using the non-parametric Wilcoxon rank sum test ( $\mathrm{p}<0.05$ was considered significant).

\section{RESULTS}

\section{Study population}

One hundred and three, of the 106 consecutive patients initially enrolled in the study, received a final diagnosis of jejunal/ileal or ileocolonic CD. Two patients were thought to suffer from indeterminate colitis, whereas one patient was classified as having chronic abdominal pain of unknown aetiology; one patient experienced severe nausea and vomiting after the ingestion of less than $250 \mathrm{ml}$ of PEG solution and was therefore excluded from the study, thus limiting the final analyses to 102 patients. All these patients underwent successful contrast enhanced US as well as complete $x$ ray and endoscopic evaluation. Nine of them (8.8\%) underwent surgery for $\mathrm{CD}$ complications and were therefore further evaluated at laparotomy. Demographic, clinical, and biological parameters of this study population are given in table I.

\section{Accuracy of conventional and contrast enhanced US in localising CD lesions}

A satisfactory distension of the intestinal lumen, as to permit a complete small bowel examination, was obtained in all patients after the ingestion of 624 (SD 52) $\mathrm{ml}$ (range $500-800 \mathrm{ml}$ ) of PEG solution with sequential visualisation

Table 1 Clinical and biochemical parameters of the $102 \mathrm{CD}$ patients enrolled in the study

\begin{tabular}{ll}
\hline Parameter & Values \\
\hline Number of females (\%) & $40(39 \%)$ \\
Age (years) & $37.3(11.8)$ \\
Disease duration (months) & $79.7(72.1)$ \\
Previous surgical resection (\%) & $30(29 \%)$ \\
Recurrences & $2.3(1.4)$ \\
CD intestinal location & $66(65 \%)$ \\
Small bowel only (\%) & $36(35 \%)$ \\
Small bowel and colon (\%) & $164.2(68.6)$ \\
CDAl (best) & $2.4(2.2)$ \\
C reactive protein (mg/ml) & $24.5(14.1)$ \\
Erithrocyte sedimentation rate (mm in the & \\
first hour) & \\
\hline Values are mean (SD) unless otherwise stated. & \\
CDAl, Crohn's disease activity index.
\end{tabular}

of jejunal and ileal loops just to the caecum. In particular, the mean time to observe the anechoic contrast flowing through the terminal ileum into the caecum (which was considered the optimal time to start a retrograde US examination of the small bowel) was 31.4 (SD 10.9) minutes (range 20-60 minutes). No major complaints were reported during or immediately after PEG ingestion, apart from mild to moderate nausea in seven patients and slight abdominal distension in four.

Overall sensitivity of conventional and contrast enhanced US in detecting $\mathrm{CD}$ lesions using barium enteroclysis and/or ileocolonoscopy as gold standard was $91.4 \%$ (CI 89.9 to 94.3 ) and $96.1 \%$ (CI 94.1 to 98.9 ), respectively. The segment-bysegment analysis revealed that contrast enhanced US had a superior overall accuracy compared with conventional US in detecting small bowel CD (table 2); indeed, sensitivity of conventional US for lesions located in the jejunum was $80 \%$ compared with $100 \%$ of contrast enhanced US. Also, in the detection of ileal abnormalities the addition of anechoic oral contrast further increased the already high sensitivity of bowel US (sensitivity increased from $92.0 \%$ to $95.9 \%$ ). In this regard, it is worth emphasising that sensitivity of contrast enhanced US in diagnosing proximal ileal diseases was quite similar to that registered for $\mathrm{CD}$ lesions located in the distal ileum.

A good comparison between conventional US, contrast enhanced US, and BE in revealing typical CD ileal lesions (such as cobblestones, ulcerations) is reported in figure l. It is worth noting that three out of four ileal lesions missed by conventional US were minor mucosal/submucosal abnormalities (scattered aphtoid ulcers and tiny irregularities of intestinal mucosa involving short tracts of the terminal ileum) two of which, however, were depicted by contrast enhanced US. In order to establish the ability of the two US techniques to assess disease extent within the small bowel, a correlation between CD extent measured at conventional and contrast enhanced US and that determined by BE was made in those patients in whom an accurate measurement of the disease length was feasible at $x$ ray. Conventional US measurement correlated well with $x$ ray involvement $(r=0.83)$, but measurements made at contrast enhanced US proved to be even more accurate $(r=0.94)$; indeed, the extent of ileal disease as evaluated by the latter technique was quite similar to that measured at $x$ ray (16.8 (SD 9.3) $v$ 19.8 (SD 11.9) cm) (fig 2).

Moreover, overall agreement $(\mathrm{k})$ of conventional and oral contrast US in detecting CD lesion sites were 0.78 (CI 0.66 to 0.89 ) and 0.89 (CI 0.78 to 0.99 ), respectively.

\section{Detection of luminal complications at conventional and contrast enhanced US}

One or more small bowel strictures were diagnosed by barium enteroclysis in 27 CD patients; conventional bowel US correctly detected the presence of strictures in 20 of these patients, with five false positive results (sensitivity $74 \%$,

Table 2 Sensitivity at segment-by-segment analysis of conventional and contrast enhanced bowel US in the detection of small bowel $C D$ lesions documented at $x$ ray/ endoscopy

\begin{tabular}{lll}
\hline $\begin{array}{l}\text { Site of lesion ascertained } \\
\text { at } x \text { ray/endoscopy }\end{array}$ & $\begin{array}{l}\text { Conventional US } \\
\text { Sensitivity } \\
(95 \% \mathrm{CI})\end{array}$ & $\begin{array}{l}\text { Contrast enhanced } \\
\text { US Sensitivity } \\
(95 \% \mathrm{CI})\end{array}$ \\
\hline Jejunum $(\mathrm{n}=5)$ & $80.0 \%(78.0-82.0)$ & $100 \%$ \\
lleum $(\mathrm{n}=98)$ & $92.0 \%(89.7-94.3)$ & $98.5 \%(96.2-100)$ \\
$\quad \begin{array}{l}\text { Proximal ileum }(\mathrm{n}=16) \\
\text { Distal ileum }(\mathrm{n}=82)\end{array}$ & $93.7 \%(91.3-96.0)$ & $93.7 \%(91.3-96.0)$ \\
\hline
\end{tabular}




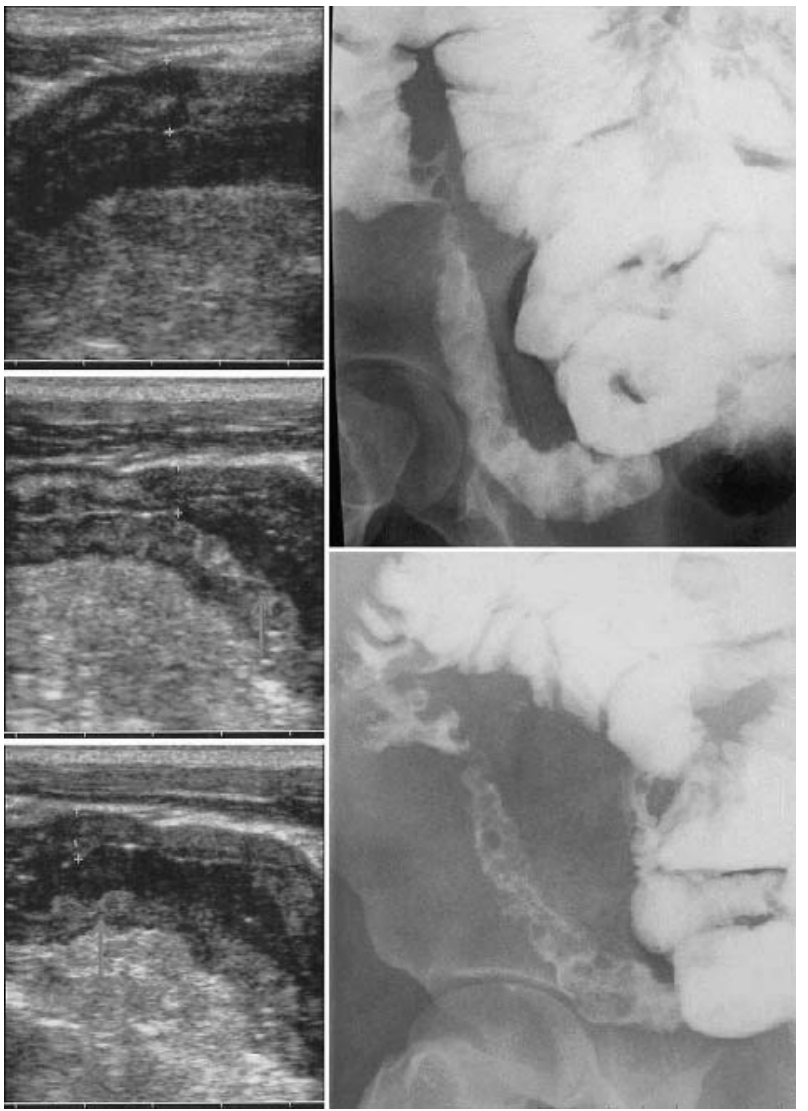

Figure 1 Oral contrast US (left) and barium enteroclysis (right) in a patient with Crohn's ileitis. Note at US longitudinal sections (from the top to the bottom) the anechoic contrast flow through the terminal ileum and the distension of bowel walls with a clear definition of the cobblestone mucosal pattern (white arrows) as well as the reduction of BWT from $8.1 \mathrm{~mm}$ to $6.2 \mathrm{~mm}$.

specificity 93\%). Distension of the lumen by anechoic contrast solution significantly increased the accuracy of US in identifying small bowel strictures (sensitivity increased to $89 \%$, and PPV to $92 \%$ ), presumably by favouring dilatation of prestenotic jejunal/ileal loops and thus permitting a better visualisation of the narrowed segment (table 3). Indeed, in the hands of the most expert sonographer (FP), agreement between US and barium enteroclysis in detecting strictures increased from 0.72 (CI 0.48 to 0.83 ) to 0.95 (CI 0.87 to 1.02 ) when shifting from conventional to contrast enhanced US.

Even the well known gap of sensitivity between the detection of a single stricture and multiple strictures at US was ameliorated by the use of oral contrast; indeed, two or more strictures were detected in $78 \%$ of patients with ascertained multiple stenoses at BE by contrast enhanced US in comparison with only 55\% depicted by conventional US.

As expected, both conventional and contrast enhanced US were more accurate in the detection of internal fistulas/sinus tracts than was BE using intraoperative findings as gold standard. Indeed, conventional US, contrast enhanced US, and BE detected $80 \%, 86 \%$, and $67 \%$, respectively, of fistulas/sinus tracts which were subsequently found at surgery. No significant difference in terms of accuracy was observed between the two US techniques with reference to this type of complication.

\section{Interobserver agreement at conventional and contrast enhanced US}

Agreement (k) between investigators for sites of CD lesions at conventional US was 0.91 (CI 0.83 to 0.99 ), and k 0.95 (CI
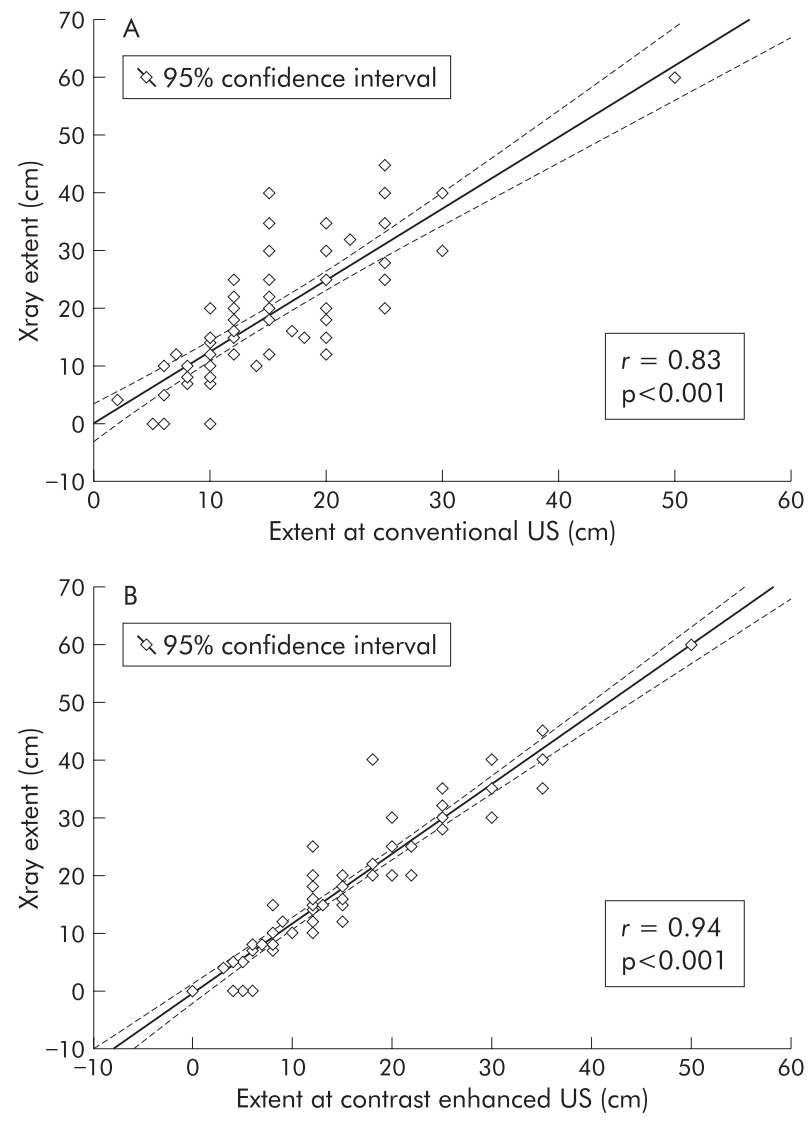

Figure 2 Correlation between conventional US and $x$ ray extent (top), and between disease extent measured at contrast enhanced US (bottom) and $x$ ray in 62 patients with ileal CD localisation only. Statistical significance between $r^{1}$ and $r^{2} p<0.01$.

0.89 to 1.00 ) at contrast enhanced US. Regarding maximum BWT measurement, a considerable difference in mean bowel thickness between the two sonographers was found at conventional US $(7.48 v 6.58 \mathrm{~cm})$. After oral contrast a significant improvement in agreement between sonographers was evident (mean BWT found by the two sonographers reduced to 7.13 and $6.47 \mathrm{~cm}$, respectively), with a mean improvement in difference of $0.13 \mathrm{~cm}$ (Student's $t$ test $=2.56, \mathrm{p}=0.01$ ).

Evaluation of elasticity of the affected bowel segments There was a significant difference in the degree of wall elasticity as judged by both sonographers between conventional and contrast enhanced US. In particular, at conventional US the mean elasticity score was 1.37 and 1.41 for the two sonographers respectively, whereas at oral contrast enhanced US the mean score increased to 2.14 and 2.11 $(p<0.05)$, respectively. A good example of an ileal loop which was thought to be stiffened and hypocontractile at conventional US, but proved to possess a well preserved elasticity after the administration of anechoic oral contrast solution, is shown in figure 3.

\section{DISCUSSION}

The radiographic examination of the small intestine with barium either as enteroclysis or as small bowel follow through are still the mainstays in small bowel imaging of patients with already known or suspected CD. ${ }^{19-21}$ Both examinations are employed in daily clinical practice in the initial evaluation of patients for the purpose of establishing diagnosis, in the preoperative setting to verify the full extent of disease, and during clinical exacerbations to determine if 
Table 3 Sensitivity, specificity, positive (PPV) and negative (NPV) predictive value of conventional bowel US and contrast enhanced bowel US in detecting one or more small bowel strictures as identified at barium $x$ ray

\begin{tabular}{|c|c|c|c|c|c|c|c|c|}
\hline & $\mathrm{TP}$ & $\mathrm{TN}$ & FP & $\mathrm{FN}$ & $\begin{array}{l}\text { Sensitivity } \\
(95 \% \mathrm{CI})\end{array}$ & $\begin{array}{l}\text { Specificity } \\
(95 \% \mathrm{CI})\end{array}$ & PPV & NPV \\
\hline \multicolumn{9}{|l|}{ Conventional bowel US } \\
\hline $\begin{array}{l}\text { At least one small bowel } \\
\text { stricture* }^{*}\end{array}$ & 20 & 70 & 5 & 7 & $\begin{array}{l}74.0 \% \\
(72.1-75.8)\end{array}$ & $\begin{array}{l}93.3 \% \\
(90.9-95.6)\end{array}$ & $80.0 \%$ & $90.9 \%$ \\
\hline $\begin{array}{l}\text { Multiple strictures (two or } \\
\text { more) }\end{array}$ & 5 & 86 & 5 & 4 & $\begin{array}{l}55.5 \% \\
(54.1-56.8)\end{array}$ & $\begin{array}{l}95.5 \% \\
(93.1-97.8)\end{array}$ & $50.0 \%$ & $95.5 \%$ \\
\hline \multicolumn{9}{|l|}{ Contrast enhanced US } \\
\hline $\begin{array}{l}\text { At least one small bowel } \\
\text { stricture* }^{*}\end{array}$ & 24 & 73 & 2 & 3 & $\begin{array}{l}88.8 \% \\
(86.5-91.0)\end{array}$ & $\begin{array}{l}97.3 \% \\
(94.8-99.7)\end{array}$ & $92.3 \%$ & $96.0 \%$ \\
\hline $\begin{array}{l}\text { Multiple strictures (two or } \\
\text { more) }\end{array}$ & 7 & 89 & 2 & 2 & $\begin{array}{l}77.7 \% \\
(75.7-79.6)\end{array}$ & $\begin{array}{l}97.8 \% \\
(95.3-100)\end{array}$ & $77.7 \%$ & $97.8 \%$ \\
\hline \multicolumn{9}{|c|}{$\begin{array}{l}\text { TP, true positive; TN, true negative; FP, false positive; FN, false negative. } \\
\text { *In this analysis a result was considered to be truly positive when at least one stricture, identified at US, was } \\
\text { matched to a lesion seen at small bowel enteroclysis. In other words, each patient was classified simply as a carrier } \\
\text { of small bowel stenosis if at least one of these lesions was revealed at } x \text { ray. }\end{array}$} \\
\hline
\end{tabular}

luminal complications (namely, strictures and fistulas) are present. Over the last few years, however, cross sectional imaging techniques such as US, CT, or magnetic resonance imaging (MRI) have proved to be able to show segmental thickening of the bowel wall, stenosis or prestenotic dilatation, as well as extraluminal lesions such as fistulas and abscesses and have, at least in part, already replaced barium studies in specialised centers dedicated to the management of inflammatory bowel disease. ${ }^{22}$

Among these techniques, bowel US probably constitutes the most attractive first choice imaging modality in terms of cost effectiveness. Because of the lack of radiation exposure, its ready availability, and low cost it can be repeated frequently to assess disease extension and severity. Over the last decade, the diagnostic value of bowel US in patients with CD has been evaluated in several studies which have shown a high correlation of this technique with the conventional radiological methods, surgery, or endoscopy. ${ }^{1-6} 1718$
Unfortunately, conventional bowel US is less sensitive than $\mathrm{BE}$ in detecting subtle $\mathrm{CD}$ lesions as they may not cause significant bowel wall thickening and the bowel is usually in a collapsed state, thus not permitting the detection of focal thickenings. For the same reason, mild to moderate luminal complications (namely, low grade strictures) tend to be underestimated by conventional US which, by contrast, is very accurate in the diagnosis of complete and high grade obstruction. ${ }^{17}$ Interobserver variability between sonographers may constitute another limit of conventional bowel US, which is a highly operator dependent technique requiring great experience and time commitment to achieve accuracy rates comparable with those reported in the literature. ${ }^{23}$

The recent availability of oral non-absorbable, isotonic, anechoic solutions able to distend the small bowel lumen and thus improving visualisation of intestinal loops, reducing air artifacts which hinder sonographic reflection, may favour the
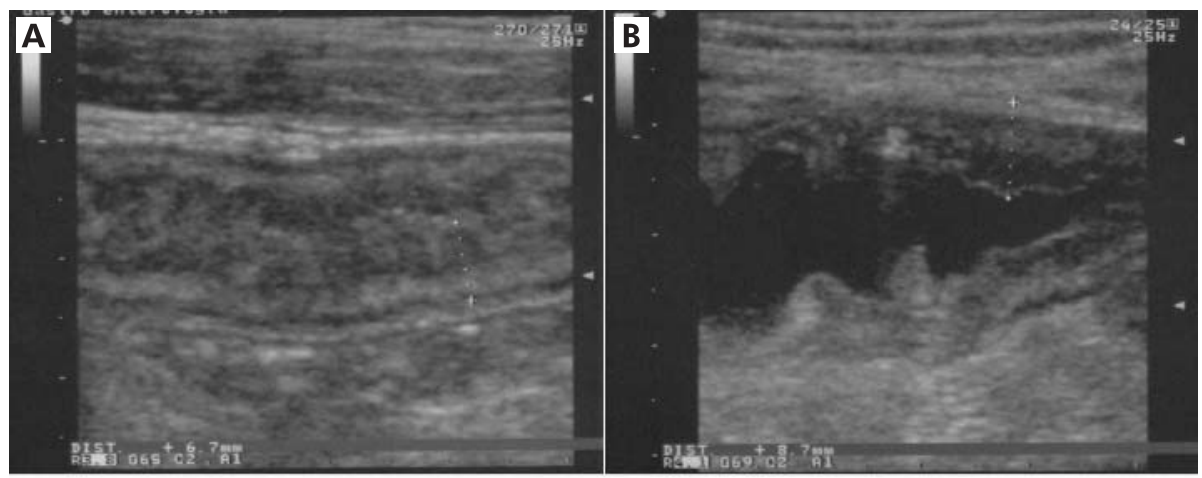

Figure 3 (A) A thickened ileal loop which seems to be stiffened and hypocontractile at conventional US longitudinal section. On the contrary, the ingestion of anechoic contrast reveals good distensibility of the bowel walls $(B, C, D)$, clearly depicting typical cobblestone appearance of the luminal surface (C).

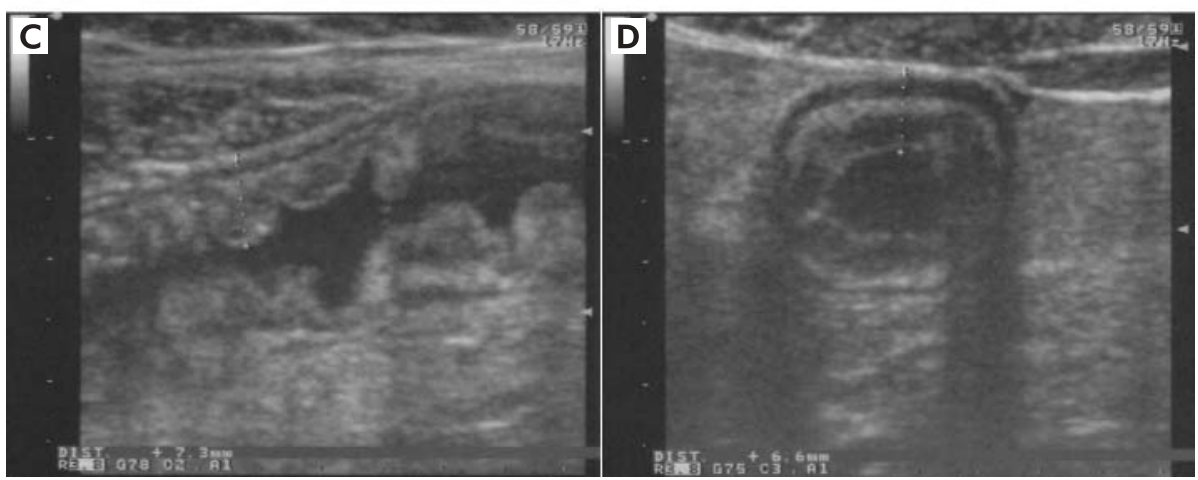


analysis of wall layers and the topographic localisation of abnormal findings, as documented recently. ${ }^{11-13}$

The present study is the first in a large series of consecutive patients that compares the accuracy of conventional US with oral contrast enhanced US in the diagnosis of CD and its related luminal complications. Our results show that the addition of an anechoic oral contrast increased the sensitivity of bowel US from $91.4 \%$ to $96.1 \%$ in the detection of small bowel CD lesions, comparing favourably with $\mathrm{BE}$; in particular, there was only one false negative diagnosis with contrast enhanced US, compared with five of conventional US and one of BE. Both these false negative examination findings were due to minimal inflammation because endoscopy demonstrated only few aphthous ulcerations in the terminal ileum. In addition, contrast enhanced US was more accurate than conventional US and almost similar to BE in measuring the extension of small bowel involvement. Moreover, the distension of intestinal lumen by anechoic contrast solution significantly increased the accuracy of US in the detection of small bowel strictures which increased in the hands of the two sonographers from $74 \%$ to $89 \%$ and from $72 \%$ to $86 \%$, respectively-presumably thanks to a more marked dilation of the prestenotic segment and a better visualisation of the narrowed tract. As the sensitivity of conventional bowel US in this setting was quite similar to that previously reported in a larger series by our group ${ }^{17}$, the increased accuracy of post distension US in stricturing CD suggests that this technique is highly reliable in detecting this type of complication.

Even the well known gap of sensitivity between the sonographic detection of a single stricture and multiple strictures (US has proved to be fairly accurate in depicting stricturing diseases but not in identifying the number of strictures within the small bowel ${ }^{17}$ was reduced by the addition of oral contrast; indeed, as many as $78 \%$ of $C D$ patients with more than one strictures at BE were identified as carrier of multiple small bowel strictures at contrast enhanced US.

As far as concerns the tolerability and the duration of the whole examination, these are highly satisfactory in comparison with $\mathrm{BE}$ or other techniques such as CT enterography, ${ }^{24}$ or MRI enteroclysis. ${ }^{25}$ In fact, the small ingested volume and the palatability of Klean-prep makes this procedure well accepted by patients, as documented by the fact that only one of the 103 cases was unable to drink enough solution because of side effects. Moreover, the cost of the product is very low (less than $€ 3.00$ per examination) which combined to the relatively low cost of US examination makes this procedure far less expensive than BE, CT, or MRI. After standardisation of the procedure, the mean duration of the whole examination is somewhat less than 20 minutes, as the post contrast phase can be started 20 minutes after PEG ingestion and completed in approximately 15 minutes. The procedure may occasionally take more time to be completed under specific circumstances, such as in the presence of multiple strictures where a careful evaluation of the entire small bowel is required in order to correctly localise their site, degree, and extension.

In conclusion, the findings emerging from the present study show that in the assessment of patients known or suspected to have CD, oral contrast enhanced bowel US performs as well as fluoroscopic small bowel examination in the detection of lesions and luminal complications, also providing important additional information on the surrounding mesentery. Thanks to the lack of radiation exposure, low cost, ready availability, and repeatability, this technique may be considered in every day clinical practice as a first choice examination in the diagnostic work up and follow up of CD patients, thus limiting the use of BE to a few selected cases.

\section{Authors' affiliations}

F Parente, S Greco, M Molteni, A Anderloni, G Bianchi Porro,

Department of Gastroenterology, L Sacco University Hospital, Milan, Italy

G M Sampietro, P G Danelli, Department of General Surgery, L Sacco University Hospital, Milan, Italy

R Bianco, Radiology Unit, L Sacco University Hospital, Milan, Italy S Gallus, Istituto di Ricerche Farmacologiche Mario Negri, Section of Clinical Epidemiology, Milan, Italy

This paper was presented at the Annual Meeting of the British Society of Gastroenterology (Glasgow, UK, 21-24 March 2004) and published in abstract form (Gut 2004:53(suppl III):A1).

\section{REFERENCES}

1 Sonnenberg A, Erckenbrecht J, Peter P, et al. Detection of Crohn's disease by ultrasound. Gastroenterology 1992;83:430-4.

2 Schwerk WB, Beckh K, Raith M. A prospective evaluation of high resolution sonography in the diagnosis of inflammatory bowel disease. Eur J Gastroenterol Hepatol 1992:4:173-82.

3 Sheridan MB, Nicholson DA, Martin DF. Transabdominal ultrasonography as the primary investigation in patients with suspected Crohn's disease or recurrence: a prospective study. Clin Radiol 1993;48:402-4.

$4 \operatorname{Lim} \mathrm{JH}, \mathrm{Ko}$ YT, Lee DH, et al. Sonography of inflammatory bowel disease: findings and value in differential diagnosis. Am J Roentgenol 1994; 163:343-7

5 Maconi G, Parente F, Bollani S, et al. Abdominal ultrasound in the assessment of extent and acitivity of Crohn's disease. Clinical significance and implication of bowel wall thickening. Am J Gastroenterol 1996;91:1604-9.

6 Gasche C, Moser G, Turetschek K, et al. Transabdominal bowel sonography for the detection of intestinal complications of Crohn's disease. Gut 1999:44:112-17.

7 Carlson HC. The small bowel examination in the diagnosis of Crohn's disease. Am J Roentgenol 1986; 147:63-5.

8 Chemish SM, Maglinte DD, O'Connor K. Evaluation of the small intestine by enteroclysis for Crohn's disease. Am J Gastroenterol 1992;87: 696-701.

9 Bernstein CN, Boult IF, Greenberg HM, et al. A prospective comparison between small bowel enteroclysis and small bowel follow-through in Crohn's disease. Gastroenterology 1997;113:390-8.

10 Parente F, Maconi G, Bianchi Porro G. Bowel ultrasound in Crohn's disease. Current role and future application. Scand J Gastroenterol 2002;37:871-6.

11 Folvik G, Bierke-Larssen T, Odergaard S, et al. Hydrosonography of the small intestine: comparison with radiologic barium study. Scand J Gastroenterol 1999;34:1247-52

12 Pallotta N, Baccini F, Corazziari E. Small intestine contrast ultrasonography (sicus) in the diagnosis of small intestine lesions. Ultrasound Med Biol $2001 ; 27: 335-41$

13 Cittadini G, Giasotto V, Garlaschi G, et al. Transabdominal ultrasonography of the small bowel after oral administration of a nonabsorbable anechoic solution:comparison with barium enteroclysis. Clin Radiol 2001;56:225-30.

14 Malchow H, Ewe K, Brandes JW, et al. European cooperative Crohn's disease Study (ECCDS): results of drug treatment. Gastroenterology 1984;86:249-66.

15 Best WR, Becktel JM, Singleton JW. Rederived values of the eight coefficients of Crohn's disease activity index (CDAl). Gastroenterology 1979;77:843-6.

16 Parente F, Greco S, Molteni M, et al. Role of early ultrasound in detecting inflammatory intestinal disorders and identifying their anatomical location within the bowel. Aliment Pharmacol Ther 2003;18:1009-16.

17 Parente F, Maconi G, Bollani S, et al. Bowel ultrasound in assessment of Crohn's disease and detection of related small bowel strictures: a prospective comparative study versus $x$-ray and intraoperative findings. Gut 2002;50:490-5.

18 Maconi G, Sampietro GM, Parente F, et al. Contrast radiology, computed tomography and ultrasonography in detecting internal fistulas and intraabdominal abscesses in Crohn's disease: a prospective comparative study. Am J Gastroenterol 2003;98:1545-55.

19 Maglinte DD, Chemish SM, Kelvin FM, et al. Crohn's disease of the small intestine: accuracy and relevance of enteroclysis. Radiology 1995; 184:541-5

20 Kelvin FM, Maglinte DD. Enteroclysis or small bowel follow-through in Crohn's disease ? Gastroenterology 1998;114:1349-51.

21 Toms AP, Barltrop A, Freeman AH. A prospective randomised study comparing enteroclysis with small bowel follow-through examinations in 244 patients. Eur Radio 2001;11:1155-60.

22 Scholmerich J. Inflammatory bowel disease. Endoscopy 2003;35:164-70.

23 McLean A. Transabdominal bowel sonography in Crohn's disease. Gut 1999;44:6-7.

24 Wold PB, Fletcher JG, Johnson CD, et al. Assessment of small bowel Crohn disease: noninvasive peroral CT enterography compared with other imaging methods and endoscopy-feasibility study. Radiology 2003;229:275-81.

25 Schreyer AG, Herfarth H, Kikinis R, et al. 3D modeling and virtual endoscopy of the small bowel based on magnetic resonance imaging in patients with inflammatory bowel disease. Invest Radiol 2002;37:528-33. 